Check for updates

Cite this: RSC Adv., 2019, 9, 31255

Received 23rd July 2019

Accepted 26th September 2019

DOI: 10.1039/c9ra05681e

rsc.li/rsc-advances

\section{Waste spunlaced facial puff derived monolithic flexible carbon framework (WCF): an ultralow-cost, recyclable and eco-friendly sorbent for oils and organic solvents $\dagger$}

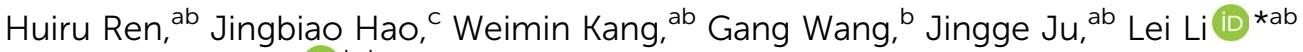 \\ and Bowen Cheng (D) bd
}

\begin{abstract}
Due to the spunlaced effect, waste spunlaced facial puff has a high degree of fiber entanglement and an abundant three-dimensional (3D) network porous structure, which make it form a 3D carbon framework material more easily after carbonization. For the first time, the monolithic 3D carbon framework is synthesized from waste spunlaced facial puff (WCF) and used as the adsorbent for contaminants in water. The adsorption capacity of WCF for oils and organic reagents can be 34-137 times its own weight. Over five adsorption-harvesting cycles, the adsorption capacity of WCF to organic pollutants can recover up to $95 \%$ of its initial capacity. Moreover, WCF exhibits stable permeation flux and high separation efficiency in a water-heavy oil system, which is about $7714 \mathrm{~L} \mathrm{~m}^{-2} \mathrm{~h}^{-1}$ and higher than $99 \%$, respectively. With a combination of waste spunlaced facial puff with monolithic 3D porous structure as a raw material, facile and green preparation process, low density, excellent hydrophobicity and lipophilicity, WCF as an adsorbent has great superiority in removal of organic pollutant solvents and environmental protection as well as other applications, such as energy storage materials, catalyst carriers, electric information, etc. Furthermore, this work would provide a new strategy for recovery use of waste spunlaced cotton materials.
\end{abstract}

\section{Introduction}

Waste cotton materials have attracted great attention in upgrading recycling due to their unique characteristics, such as natural curls, high surface area, numerous active hydroxyl groups and the main component of cellulose as a carbon precursor. ${ }^{1,2}$ Initially, waste cotton-based materials were normally used in some traditional areas, including production of clothing, geotextiles, carpets, nanoparticles and composite materials..$^{3-8}$ Recently, to further broaden their application field and improve their application value, the waste cotton materials are converted into carbon-based materials via carbonization or other methods, and are being applied in some new fields, e.g. secondary battery electrodes, ${ }^{9-11}$ strain sensors and contaminant adsorbents. ${ }^{12-20}$

${ }^{a}$ School of Textile Science and Engineering, Tiangong University, 300387, P. R. China. E-mail: lileil_003@126.com

${ }^{b}$ State Key Laboratory of Separation Membranes and Membrane Processes, Tiangong University, 300387, P. R. China

${ }^{c}$ Nox Bellcow Cosmetics Co., Ltd., 528427, P. R. China

${ }^{d}$ Tianjin University of Science \& Technology, 300222, P. R. China

$\dagger$ Electronic supplementary information (ESI) available. See DOI: 10.1039/c9ra05681e
Over the last decades, water pollution triggered by oil spillage and emissions of toxic organic solvents have become an urgent global issue, which are greatly harmful to the environment and ecology. ${ }^{21}$ Adsorption is considered to be the most promising solution to solve the oil pollution problem due to its high efficiency, ease of operation and recyclability. ${ }^{22,23}$ Thereinto carbon-based materials including active carbons, ${ }^{24}$ carbon nanoparticles ${ }^{25}$ and carbon nanofibers ${ }^{26}$ have been widely researched as oil and organic solvent adsorbents because of their lipophilic inherent nature. Moreover, in order to further improve their adsorption performance, the 3D carbon frameworks such as carbon foams, ${ }^{27-30}$ carbon sponges $^{31-35}$ and carbon aerogels ${ }^{36-39}$ via physical/chemical assembly of carbonbased building blocks (e.g. carbon nanotubes, graphene/ graphite nanosheets and so on) have captured more and more attentions because of their light weight and high sorption capacity. The carbon-based 3D frameworks synthesized through this route usually exhibit a high sorption capacity and outstanding recyclability when utilized for sorption of oils and organic solvents. However, the complex synthesis processes and high cost have hampered their massive production and practical use. Therefore, it is particularly required to develop novel 3D carbon framework materials with facile and green preparation method as well as low-cost raw materials. 
Spunlace is a fast-growing nonwoven processing method in recent years, which uses a high-pressure water jet to wind loose fiber components to endue final nonwoven fabric strength, ${ }^{40}$ thereby improving its mechanical properties and flexibility, and the resulting products present an obvious 3D network porous structure. As is well-known, facial puff is a common cotton material in our daily life, which is mainly composed of cellulose ${ }^{41}$ It is worth noting that as a typical spunlaced nonwoven product, the facial puff has some excellent characteristics, such as inherent and obvious 3D network porous structure, significantly enhanced fiber-to-fiber entanglement, and improved mechanical properties and flexibility, which is an excellent 3D carbon framework precursor material. In addition, facial puff is a kind of daily necessities for most women, it has been estimated that the global market of facial puff is up to 1.401 billion dollars in 2019, which is consumed a lot in daily life. Nevertheless, at present, the vast majority of this kind of cotton materials are discarded after use, which not only causes waste of materials, ${ }^{42}$ but also leads to great environmental pollution due to the residual chemical elements on them after use. This indicates that the disposal of discarded facial puff waste is very essential and urgently needed to receive attention.

In this paper, a novel oil and organic solvent adsorbent with 3D carbon framework is prepared by using waste spunlaced facial puff as raw material through simple pyrolysis process (WCF). Interestingly, this adsorbent not only possesses excellent flexibility, high adsorption capacity and outstanding recycle performance, but also shows remarkable oil-water separation properties. Besides, the WCF also has the potential applications in other fields, e.g. energy storage, catalyst carrier and so on. Particularly, this work would have great benefits to the waste disposal and exploitation of novel oil and organic solvent adsorbents.

\section{Experimental section}

\subsection{Materials}

Original waste spunlaced facial puffs ( $98 \%$ cellulose) were collected from daily life. Walnut corn oil, sesame oil, soybean oil and sunflower seed oil were purchased from local markets. Methanol, ethanol, isopropyl alcohol, acetone, chloroform, dichloroethane, dimethicone, pump oil, dimethylformamide, deionized water and Oil Red $\mathrm{O}$ were all purchased from Tianjin Guangfu Regent Co. LTD, which were analytical grade and without further purification.

\subsection{Preparation of 3D carbon frameworks from waste spunlaced facial puff (WCF)}

The 3D waste spunlaced facial puffs were placed into a tubular furnace for pyrolysis directly without further treatment. The heating furnace was heated up to $800{ }^{\circ} \mathrm{C}$ with a heating rate of $5{ }^{\circ} \mathrm{C} \min ^{-1}$ and kept for 2 hours under nitrogen atmosphere. Finally, the furnace was naturally cooled to room temperature to acquire the 3D carbon framework materials.

\subsection{Characterization of waste spunlaced facial puff and WCFs}

All samples were characterized by a field emission scanning electron microscope (FESEM, Hitachi S4800). Elemental analysis was accomplished by energy-dispersive spectroscope (EDS) attached to FESEM. Fourier transform infrared (FTIR) spectra were obtained on a Nicolet is50 spectrometer. For each spectrum, resolution was better than $0.09 \mathrm{~cm}^{-1}$. The contact angle (CA) was measured by a contact angle tester (JC2000D, Powereach Co., Ltd., Shanghai, China).

\subsection{Sorption of oils and organic solvents}

In a typical adsorption process, a piece of WCF (about $10 \mathrm{mg}$ ) was contacted with a kind of oil or organic liquid. The adsorbent was removed for weight measurement in a certain time later. Weight measurement should be made fast to avoid measurement errors caused by the evaporation of adsorbed volatile organic solvents. The weight of WCF before and after adsorption was measured and used to calculate the weight increase. The WCF recycling performance was tested via distillation, combustion and extrusion methods, respectively, depending on the characteristics of the adsorbates.

\subsection{Separation of oils/water}

The application of WCF in the field of oil/water separation was initially tested. The layered solution obtained by mixing water and heavy oil (chloroform dyed with Oil Red O dye) in a volume ratio of $1: 3$ was directly poured into a filter cup. WCF was fully wetted with the oil to reach the oil-absorbing saturation state, in order to accurately measure its flux and separation efficiency.

The water rejection and permeation flux were calculated by eqn (1) and (2), respectively.

$$
R(\%)=\left(1-\frac{C_{\mathrm{p}}}{C_{\mathrm{F}}}\right) \times 100 \%
$$

where: $R$ is the water rejection, $C_{\mathrm{p}}$ is the water concentration in the permeation solution and $C_{\mathrm{F}}$ is the water concentration in the feeding stream.

$$
J=\frac{V}{A T}
$$

where: $J$ is the permeation flux $\left(\mathrm{L} \mathrm{m}^{-2} \mathrm{~h}^{-1}\right), V$ is the permeation volume (L), $A$ is the effective permeation area $\left(\mathrm{m}^{2}\right)$, and $T$ is the permeation time $(\mathrm{h})$.

\section{Results and discussion}

\subsection{Synthesis and characterizations of WCF}

As mentioned, under the action of high-pressure water jets, the fibers of the spunlaced facial puff are intertwined with each other, forming a rich 3D network porous structure, which makes it easier to form 3D carbon framework material after carbonization. The 3D WCF is prepared by a simple pyrolysis process using the waste spunlaced facial puff as raw materials. Briefly, a prepared waste spunlaced facial puffs with 3D frameworks are pyrolyzed at $800{ }^{\circ} \mathrm{C}$ and kept for $2 \mathrm{~h}$ under nitrogen atmosphere to produce WCF materials (Fig. 1). 


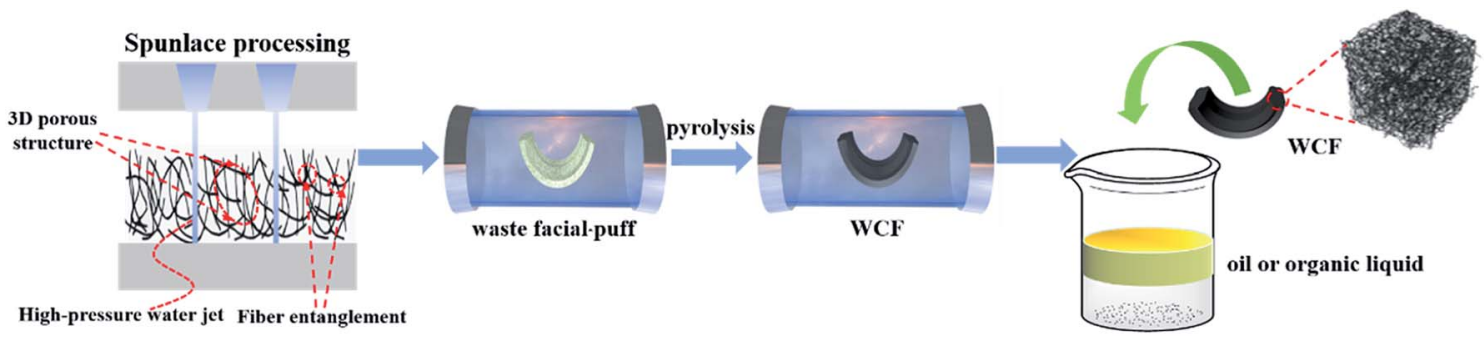

Fig. 1 Schematic diagram of the synthesis process of spunlaced facial puff and WCF.

After pyrolysis, the length of the $3 \mathrm{D}$ waste facial puff decreases from $6.04 \mathrm{~cm}$ to $4.48 \mathrm{~cm}$ (insets in Fig. 2a and b, respectively). In addition, the weight of the $3 \mathrm{D}$ waste facial puff drops from 427.06 to $97.86 \mathrm{mg}$, and the WCF has a low density of $0.0786 \mathrm{~g} \mathrm{~cm}^{-3}$. Scanning electron microscopy (SEM) image exhibits that the waste spunlaced facial puff has apparent interconnected 3D network porous structure, and the fibers are entangled with each other due to the spunlace action. Besides,
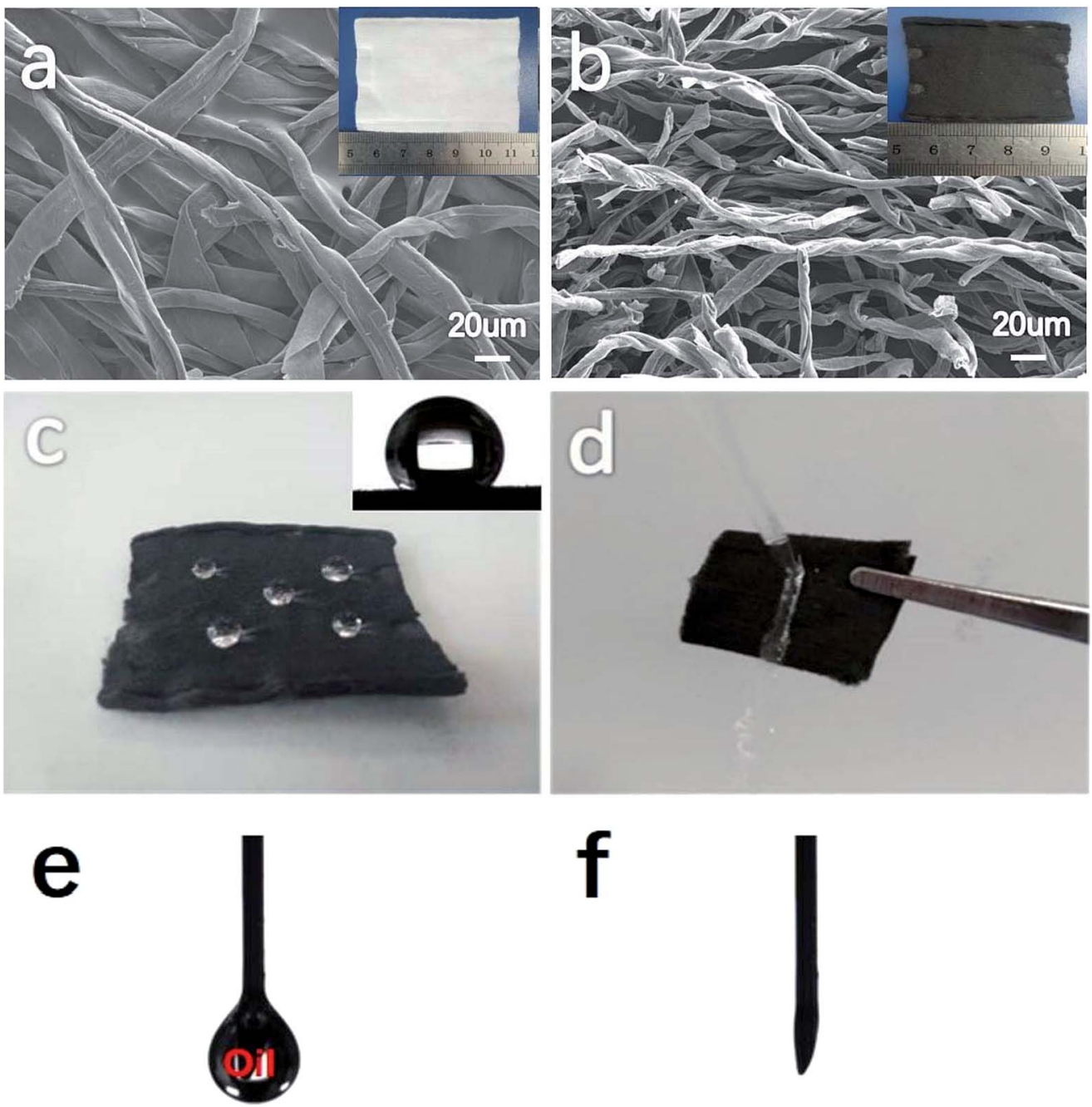

\section{Os}
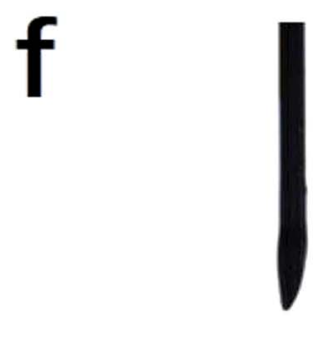

Fig. 2 (a) SEM image of the cellulose fibers in waste spunlaced facial puff and the inset is the photograph of a piece of waste spunlaced facial puff; (b) SEM image of the carbon fibers in WCF and the inset is the photograph of a piece of WCF; (c) photograph of water droplets supported on the WCF and inset is the water contact angle of WCF; (d) water flows directly down as it impacts the WCF surface. (e) and (f) Contact angle of WCF to walnut corn oil. 
the long fibers with different degrees of distortion are evenly distributed, and the fiber diameter is between 10-20 $\mu \mathrm{m}$ (Fig. 2a). In contrast, the WCF shows a smaller diameter of 5-10 $\mu \mathrm{m}$ and a further distorted form. Moreover, the WCF still remains abundant porous structure and fiber to fiber entanglement after pyrolysis process, which is beneficial to its organic pollutant adsorption performance (Fig. 2b). Although the original waste facial puff is hydrophilic, the WCF is hydrophobic with the water contact angle of $134^{\circ}$ (Fig. 2c inset) and can prop up spherical water droplets on its surface (Fig. 2c). In addition, we use water to impact WCF, and the water flows directly down the surface of WCF, as shown in the Fig. 2d. To further confirm the hydrophobicity of the WCF, the water dyed with methylene blue is dropped on the raw waste facial puff and WCF respectively (Movie S1 in ESI $\dagger$ ). The raw waste facial puff is instantly soaked by water, while the water droplets remain on the surface of WCF, showing a hydrophobic state. Then, tweezers are used to pick up the dripping waste facial puff and the WCF. The water is completely adsorbed by the raw waste spunlaced facial puff, whereas the water rolls off the surface of WCF. Furthermore, the oil contact angle of the WCF can be obtained by dropping the walnut corn oil onto the surface of the WCF. As shown in the Fig. 2e and $\mathrm{f}$, the walnut corn oil penetrates into the WCF at the moment of contact with the WCF, showing super lipophilicity of the WCF material.

Theoretically illustrates the hydrophobic and lipophilic properties of WCF. First of all, the atom compositions and distribution of waste spunlaced facial puff fibers (Fig. 3a1-a4) are characterized by EDS analysis. The EDS pattern of waste spunlaced facial puff fibers reveals that the fibers contain carbon (C), nitrogen (N) and oxygen (O) elements. The homogeneous distribution of carbon (C) and oxygen (O) elements over fibers is further characterized by element mapping images in Fig. $3 \mathrm{a} 2$ and a4. It can be seen that the distributions of elements ( $\mathrm{C}$ and $\mathrm{O}$ ) are quite uniform, which provides the elements required for hydrophilic properties. And then, the atom compositions and distribution of WCF (Fig. 3b1-b4) are also characterized by EDS analysis. It reveals that the WCF mainly contains carbon (C) elements, that means after the pyrolysis process, the oxygen $(\mathrm{O})$ elements contained in the raw waste spunlaced facial puff fibers are basically removed, and the remaining carbon is hydrophobic and oleophilic in nature. More specifically, different wettability between waste spunlaced facial puff and WCF can be demonstrated by Fourier transform infrared spectroscopy (FTIR) analysis. The FTIR spectrum of the raw waste spunlaced facial puff exhibits several hydrophilic functional group peaks, such as $\mathrm{C}-\mathrm{O}, \mathrm{C}=\mathrm{O}$ and $-\mathrm{OH}$. In contrast, all adsorption bands of hydrophilic functional groups disappear once the waste spunlaced facial puff materials are pyrolyzed, leading to its hydrophobicity and lipophilicity (Fig. S1 in ESI $\dagger$ ). Therefore, WCF materials are hydrophobic and oleophilic, and thus show potential applications in removing organic matter from water.

In addition, it is necessary and essential for WCF to have desirable flexible properties in its practical adsorption application. As shown in Fig. 4b and d, the waste spunlaced facial puff and the WCF have been folded several times, respectively, which clearly proves that the WCF has outstanding flexibility. Of course, this excellent flexibility is inseparable from the effective entanglement between the fibers of waste spunlaced facial puff. Moreover, when WCF is exposed to flame, it exhibits excellent fire resistance, which suggests its potential application under harsh high temperature condition (Movie S2 in the ESI $\dagger$ ).

\subsection{Sorption of oils and organic solvents by using WCF}

In a word, WCF has many advantages, such as good flexibility, light weight, hydrophobic and lipophilic, 3D structure, and most importantly, the raw materials of WCF are from a large amount of waste generated in daily life, energy saving and easy to prepare. The monolithic WCF's entanglement of fiber to fiber given by the spunlace process is beneficial to the adsorption. Specifically, it is convenient to separate and does not easily remain in the adsorbed liquid thus avoiding the secondary pollution. These excellent physical and chemical properties
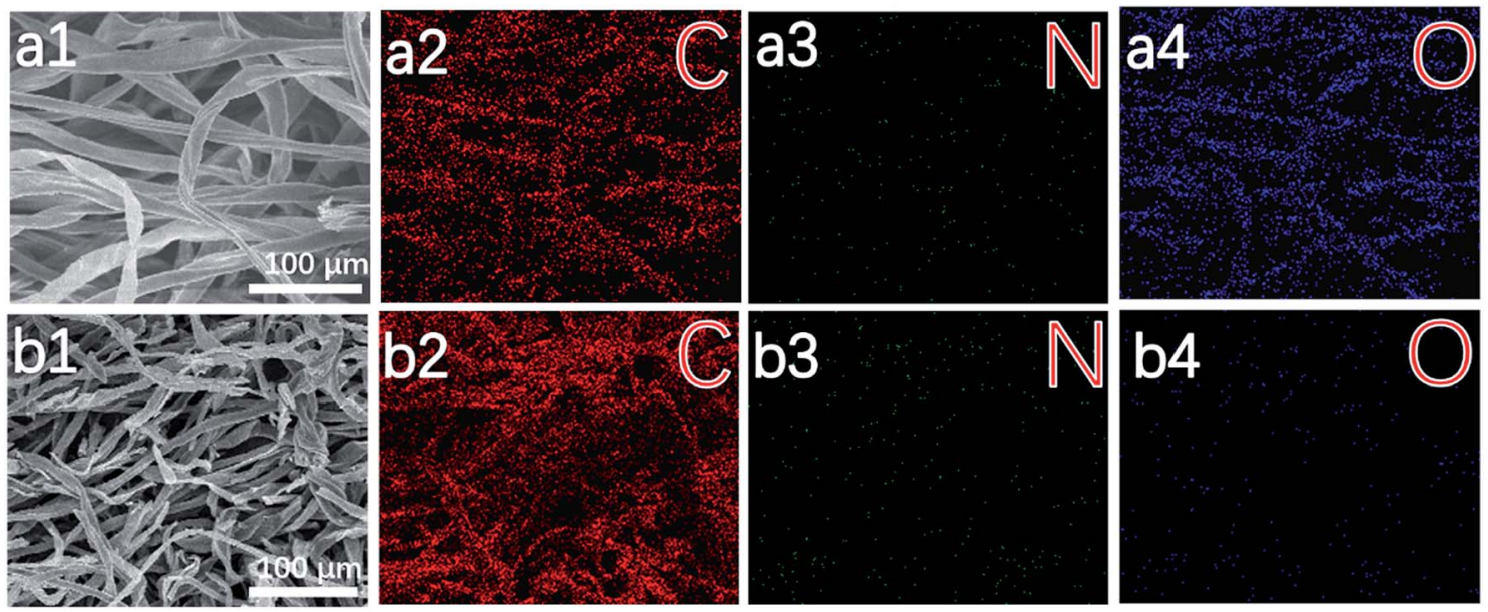

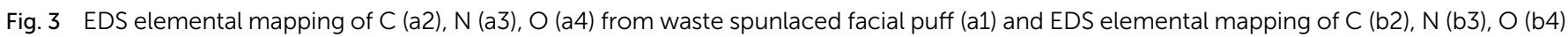
from WCF (b1). 

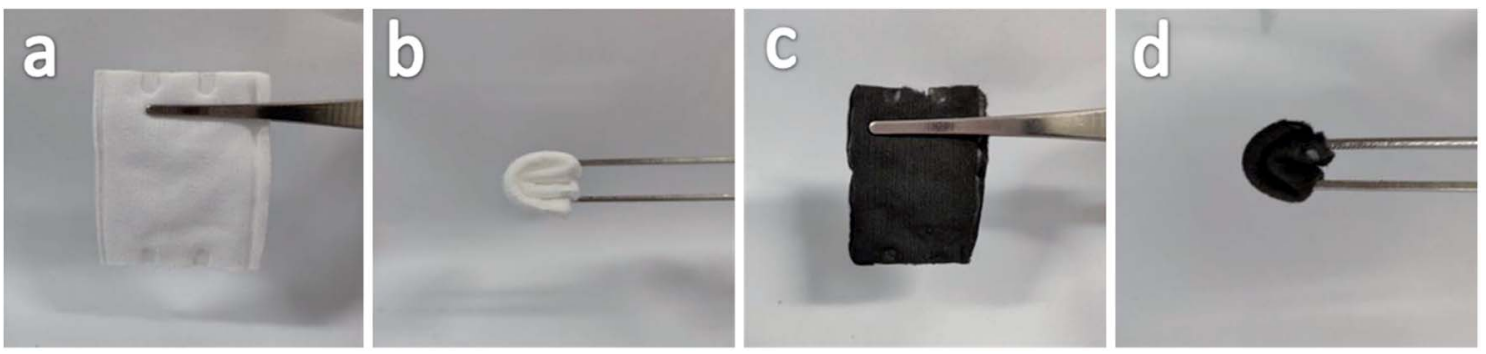

Fig. 4 (a) Photograph of a piece of waste spunlaced facia puff; (b) photograph of a piece of waste spunlaced facial puff that is folded several times; (c) photograph of a piece of WCF; (d) photograph of a piece of WCF that is folded several times.
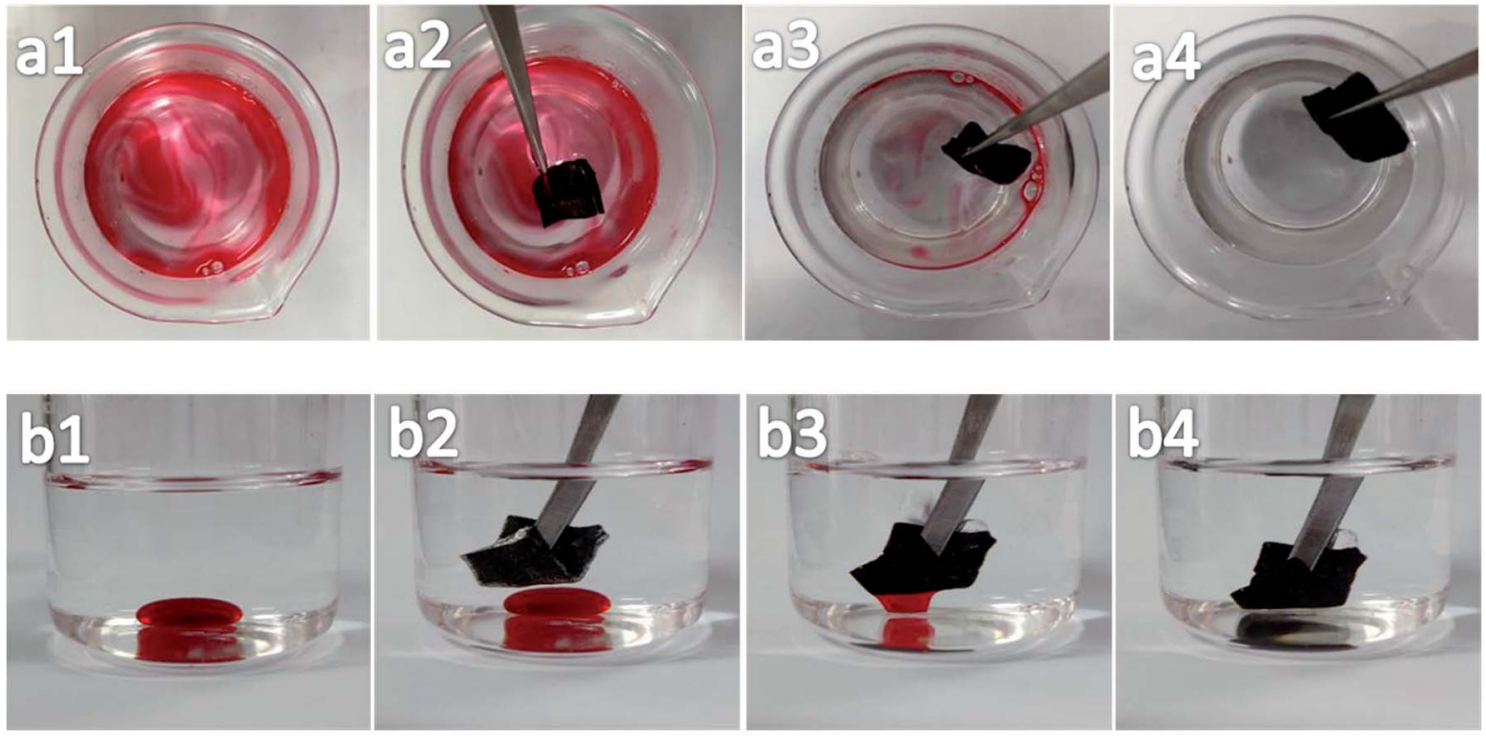

Fig. 5 Sorption of oils and organic liquids by WCF. (a) Photographs of the dimethicone sorption process at intervals of 10 s. Dimethicone stained with Oil Red O floating on water is completely adsorbed within $30 \mathrm{~s}$. (b) Photographs of the chloroform sorption process. Chloroform stained with Oil Red $\mathrm{O}$ at the bottom of water is completely adsorbed within $2 \mathrm{~s}$.

make WCF an ideal candidate for efficient removal of oils and organic solvents. The adsorption processes of oils and organic solvents using WCF are demonstrated in Fig. 5. Once the WCF is in contact with the dimethicone layer (which is stained with Oil Red O) on the water surface, the WCF can adsorb dimethicone rapidly and completely within 30 seconds (Fig. 5a). Mainly due to its hydrophobicity and low density, WCF floats on the water surface after adsorption of the dimethicone, indicating that it is easy to remove oil spills and chemical leaks, and recycle it with little effort. Furthermore, the WCF can adsorb chloroform (which is stained with Oil Red O) quickly within 2 seconds at the bottom of the water (Fig. 5b).

In order to quantitatively study the adsorption capacity, the weight increase ( $\mathrm{wt} \%)$ is defined as the weight of the adsorbate matter per unit weight of the dried WCF. A variety of oils and organic solvents are tested, including fats (e.g. sunflower oil and walnut corn oil), commercial petroleum products (e.g. pump oil) and ketones (e.g. acetone). All of them are common contaminants not only in our daily life but also from industry. Additionally, adsorption of organic liquids, including alcohols (e.g. methanol, ethanol and isopropyl alcohol), and other organic solvents (e.g. chloroform and dimethylformamide), are also studied. Obviously, the WCF exhibits a high adsorption capacity for the above oils and organics. In a word, the ability of WCF to

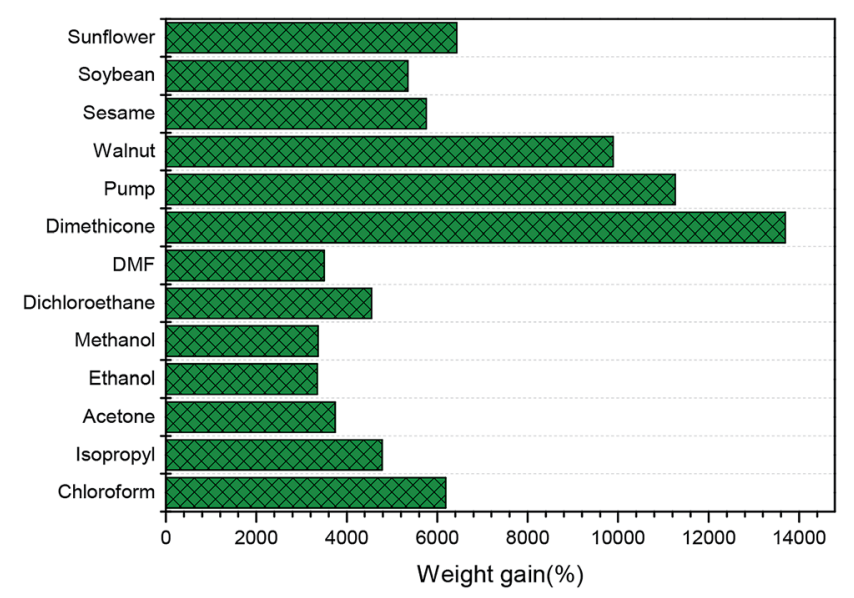

Fig. 6 Sorption efficiency of the WCF for various oils and organic liquids. 
Table 1 Comparison of various 3D sorbent materials

\begin{tabular}{llrr}
\hline Sorbent & Oils & Sorption capacity $\left(\mathrm{g} \mathrm{g}^{-1}\right)$ & Ref. \\
\hline CNF/carbon foam & Wash oil & 28 & 28 \\
UFC foam & Chloroform & 158 & 29 \\
CNF aerogels & Carbon tetrachloride & 75 & 33 \\
GSs & Diesel oil & 129 & 35 \\
Carbon aerogel & Paraffin oil & 86 & 36 \\
MTMS-DMDMS gel & Chloroform & 14 & 51 \\
Spongy graphene & Petrol oil & 86 & 48 \\
WCF & Dimethicone & 137 & This work
\end{tabular}

adsorb these liquids is up to 137 times its own weight (Fig. 6). By comparison, WCF shows a higher adsorption capacity than other previously reported materials, such as rice husks (5.02 times) ${ }^{43}$ potato peel (2.15 times) ${ }^{44}$ wool-based nonwoven (15 times), ${ }^{45}$ luffa (85 times) ${ }^{46}$ Kapok fiber $\left(58.8\right.$ times ${ }^{47}$ and spongy graphene (86 times) ${ }^{48}$ While the adsorption capacity of WCF for oils and organic solvents is a little lower than that of CNF aerogel, ${ }^{16}$ nitrogen-doped graphene foam ${ }^{49}$ and ultra-flyweight aerogels,$^{50}$ the preparation method of WCF is the simplest, and its raw materials are not only environmentally friendly biomass-based materials, which are the cheapest of all these adsorbents, but also from waste spunlaced facial puff. Hence, WCF studied in our work is a cost-effective, the most environmentally friendly and sustainable adsorbent for the removal of oils and organic contaminants. Besides, in order to better understand the adsorption performance, a table about the comparison of the adsorption performance between this material and other 3D materials is demonstrated (Table 1).
It is also necessary and essential for oil and chemical cleanup to have the recyclability of sorbents and the recoverability of contaminants. Additionally, it is also very pivotal and necessary that the adsorbent is not supposed to produce secondary pollution after adsorption. In general, three common approaches are applied to testify these properties of the sorbents for recycling, such as distillation, combustion and squeezing, which depends on the types of pollutants. More specifically, for recovering those nonflammable and precious contaminants with high boiling points, squeezing is a good choice. Combustion is suitable for the recovery of the useless and flammable pollutants, and distillation is an efficient and attractive method to remove valuable contaminants or those with low boiling points..$^{15}$ Different recycling processes of WCF are demonstrated in Fig. 7. For instance, WCF adsorbed ethanol can be efficiently removed by combustion, and the framework remains in its original form, size and inherent 3D porous structure after combustion (Fig. 7a). In contrast, walnut corn oil
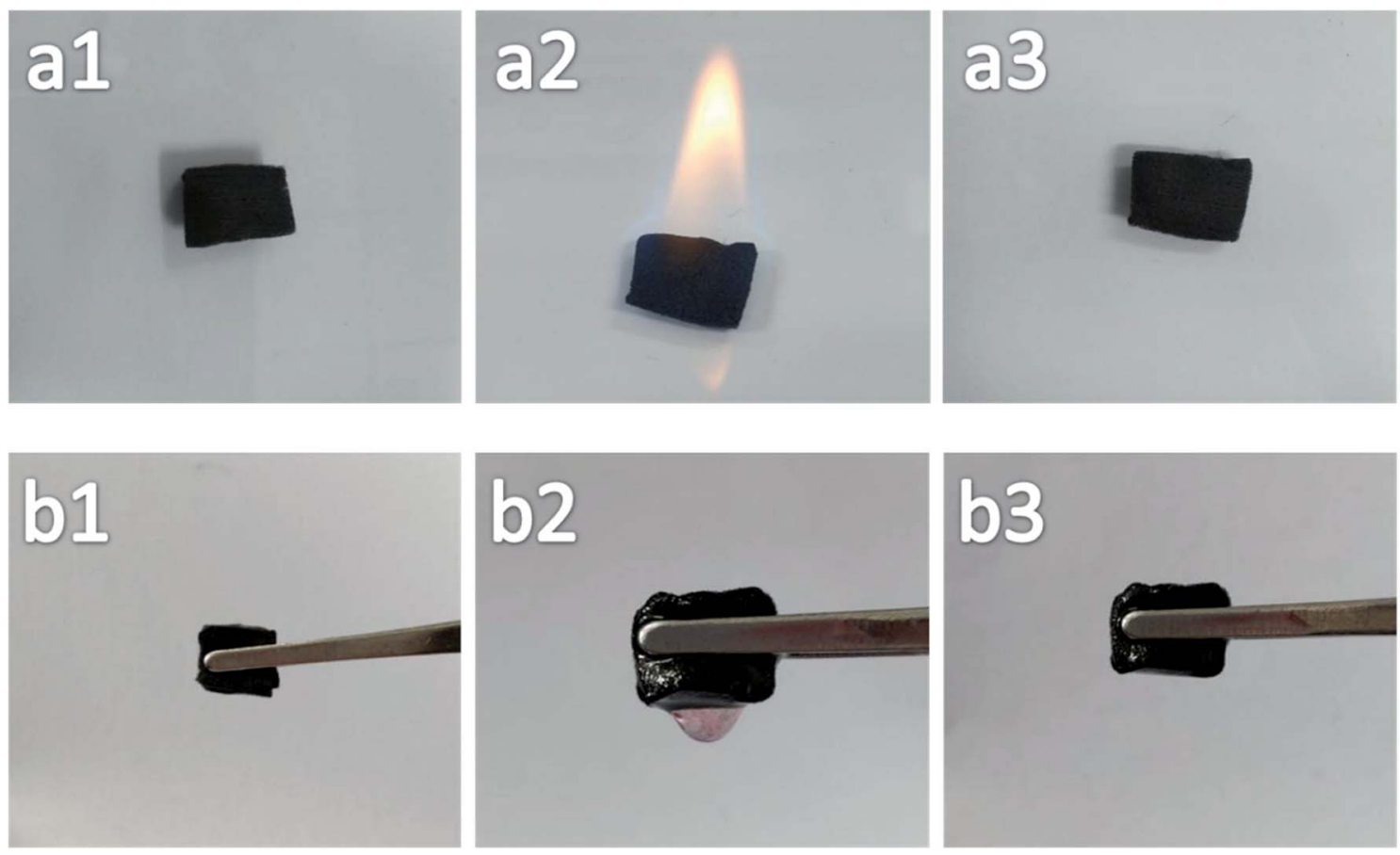

Fig. 7 (a) Photographs of the recycling process of WCF via combustion; (b) photographs of the recycling process of WCF via squeezing. 
with a high boiling point can be recovered by squeezing the WCF materials (Fig. 7b).

In addition, the WCF are subjected to repeated cycle tests through distillation (Fig. 8a), combustion (Fig. 8b) and squeezing (Fig. 8c). Initially, the method of distillation is used to test the adsorption of acetone to perform cycle test. Thereafter, the material is heated to a certain temperature to release the acetone vapor (which is chosen to evaporate at a temperature close to the boiling point of the adsorbate). The adsorptionevaporation process is repeated 5 times. As shown in Fig. 8a, after each cycle, there is almost no residual acetone in the WCF, and there is no significant change in adsorption capacity after 5 cycles, which indicates that the WCF has stable adsorption and recycling performance. Besides, no damage to the morphology and structure of the WCF is observed after several cycles. In the case of combustion recovery, ethanol is used as an example. After 5 cycles of adsorption-combustion process, the adsorption capacity of WCF is almost the same as that of the first cycle (Fig. 8b), indicating that the combustion method is also very suitable for the recycling of WCF. In addition, after repeated adsorption-combustion cycles, the 3D fiber network and twisted structure of WCF still exist. Finally, for the cyclic adsorption-extrusion test, particularly for those pollutants being nonflammable or having a higher boiling point, high boiling point dimethicone is taken as an example. In the first cycle, $972.6 \mathrm{mg}$ of dimethicone can be adsorbed by the WCF. After squeezing, due to incomplete compression of the WCF, residual dimethicone is present with a remnant mass of $264.5 \mathrm{mg}$. In order to maintain the good adsorption capacity of WCF, the strain caused by extrusion should not exceed $68 \%,{ }^{14}$ thus to retain the porous structure and twisted fiber morphology. From the second cycle, the performance of the WCF tends to stabilize, the weight gain remains unchanged (Fig. 8c). Therefore, based on its outstanding monolithic flexible framework and excellent thermal stability, the three common methods mentioned above, namely distillation, combustion and extrusion, or a combination thereof, can circulate WCF according to the type of contaminant.

What is more, the adsorption mechanism of oils and organic solvents is analyzed. The mechanism of oil-adsorbing materials for oil spill treatment is very complicated, which can be affected by various factors. ${ }^{52}$ The mechanism of oil-adsorbing materials for oil spill treatment can be divided into two types: capillary action and van der Waals interaction. Owning to the high porosity and unique pore structure caused by spunlace effect, WCF can adsorb considerable amount of organic pollutants to

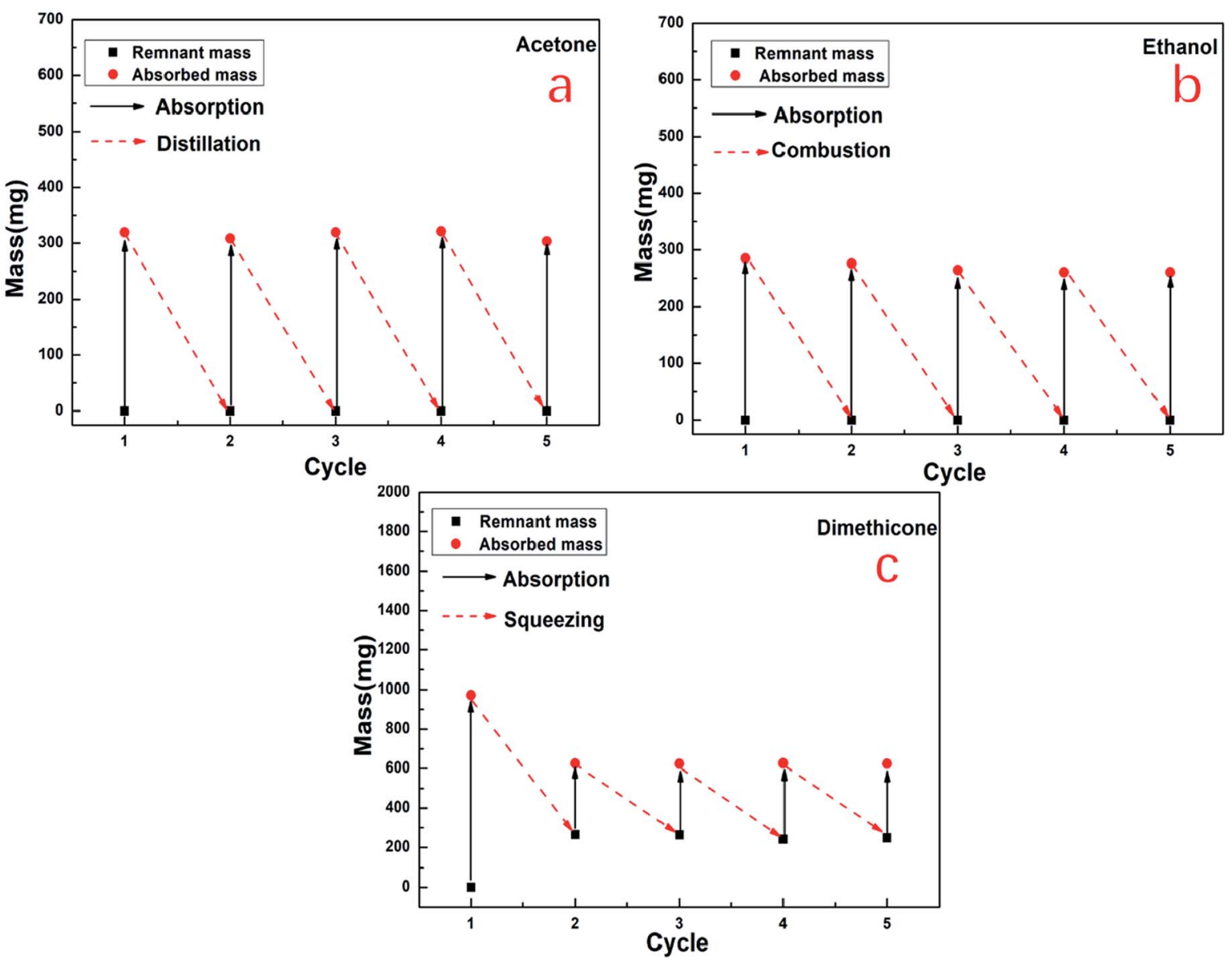

Fig. 8 (a) Distillation is applied to recycle the WCF for sorption of acetone; (b) combustion is applied to recycle the WCF for sorption of ethanol and (c) squeezing is used to recycle the WCF for sorption of dimethicone. 


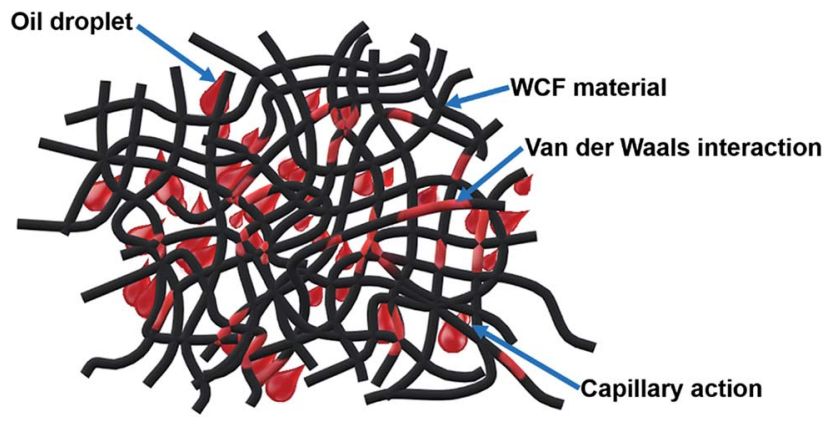

Fig. 9 Oil (dyed with Oil Red O dye) adsorption of WCF material.

its pores through capillary action. ${ }^{53,54}$ Additionally, traditional plant fiber oil-adsorbing materials have poor oil adsorption and oil retention properties due to their large amount of hydrophilic hydroxyl groups. However, after pyrolysis of the waste spunlaced facial puff, the hydrophilic functional groups are gradually lost, such as $\mathrm{C}-\mathrm{O}, \mathrm{C}=\mathrm{O}$ and $-\mathrm{OH}$, and the lipophilicity of the surface increases, the oleophilic group of WCF adsorbs the oil spilled molecules through van der Waals interaction. WCF integrates the two adsorption mechanisms (capillary action and van der Waals interaction adsorption mechanisms) to work together, which greatly enhances its ability to adsorb oil and organic pollutants, as shown in Fig. 9.

\subsection{Analysis of WCF oil/water separation performance}

Due to its flexible, abundant 3D porous structure, and excellent hydrophobic and lipophilic performances, the application of WCF in the field of oil/water separation is initially tested. The layered solution obtained by mixing water and heavy oil (chloroform dyed with Oil Red $O$ dye) in a volume ratio of $1: 3$ is directly poured into a filter cup. It is found that chloroform quickly penetrates the WCF while water remains on the upper side of the WCF. The schematic diagram of the oil/water separation process is shown in Fig. 10. The results show that WCF exhibits stable permeation flux and high separation efficiency for separating water-heavy oil system, which are about $7714 \mathrm{~L}$

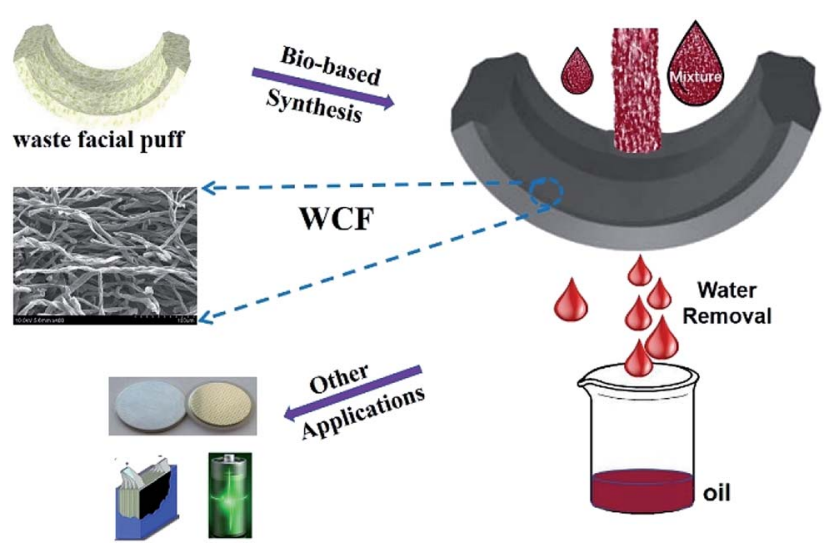

Fig. 10 Gravity-driven separation of a heavy oil (chloroform dyed with Oil Red $O$ dye) and water mixture with WCF material. $\mathrm{m}^{-2} \mathrm{~h}^{-1}$ and higher than $99 \%$, respectively. Moreover, WCF also has great potential applications in many other areas, including 3D electrode material for energy storage devices (e.g. lithium ion batteries and supercapacitors) and catalyst carrier.

\section{Conclusion}

In summary, the monolithic 3D carbon framework is synthesized from waste spunlaced facial puff (WCF) and used as the adsorbent for contaminants in water. Because of its high degree of fiber entanglement and abundant three-dimensional (3D) network porous structure, the waste spunlaced facial puff is more easily to form the $3 \mathrm{D}$ carbon framework material after carbonization. The adsorption capacity of WCF for oils and organic reagents can be 34-137 times of its own weight. Over five adsorption-harvesting cycles, the adsorption capacity of WCF to organic pollutants can recover up to $95 \%$ to its initial capacity. In addition, WCF shows stable permeation flux and high separation efficiency in water-heavy oil separating system, which are about $7714 \mathrm{~L} \mathrm{~m}^{-2} \mathrm{~h}^{-1}$ and higher than $99 \%$, respectively. Most importantly, the rich raw material of waste spunlaced facial puffs and simple pyrolysis preparation process make WCF cost-effective for practical industrial organic pollutant adsorption and separation application.

\section{Conflicts of interest}

There are no conflicts to declare.

\section{Acknowledgements}

This work was supported by the National Natural Science Foundation of China (Grant No. 51802216, No. 51678411), Natural Science Foundation of Tianjin (Grant No. 18JCQNJC76900), The Science \& Technology Development Fund of Tianjin Education Commission for Higher Education (Grant No. 2018KJ196) and State Key Laboratory of Membrane and Membrane Separation, Tiangong University.

\section{References}

1 D. Roy, M. Semsarilar, J. T. Guthrie and S. Perrier, Chem. Soc. Rev., 2009, 38, 2046-2064.

2 M. Zhang, C. Wang, Q. Wang, M. Jian and Y. Zhang, ACS Appl. Mater. Interfaces, 2016, 8, 20894-20899.

3 X. Chen, J. An, G. Cai, J. Zhang, W. Chen, X. Dong, L. Zhu, B. Tang, J. Wang and X. Wang, Polymers, 2019, 11, 404.

4 N. Pensupa, S. Y. Leu, Y. Hu, C. Du, H. Liu, H. Jing, H. Wang and C. S. K. Lin, Top. Curr. Chem., 2017, 375, 76.

5 S. Montava-Jordà, S. Torres-Giner, S. Ferrandiz-Bou, L. Quiles-Carrillo and N. Montanes, Int. J. Mol. Sci., 2019, 20, 1378.

6 P. Peña-Pichardo, G. Martínez-Barrera, M. Martínez-López, F. Ureña-Núñez and J. M. L. dos Reis, Constr. Build. Mater., 2018, 177, 409-416.

7 F. Quartinello, S. Vecchiato, S. Weinberger, K. Kremenser, L. Skopek, A. Pellis and G. Guebitz, Polymers, 2018, 10, 1107. 
8 A. Serra, Q. Tarrés, M. Llop, R. Reixach, P. Mutjé and F. X. Espinach, Text. Res. J., 2019, 89, 2113-2125.

9 C. Wei, J. Yu, X. Yang and G. Zhang, Nanoscale Res. Lett., 2017, 12, 379.

10 B. Zheng, N. Li, J. Yang and J. Xi, Chem. Commun., 2019, 55, 2289-2292.

11 Z. Wang, D. Xue, H. Song, X. Zhong, J. Wang and P. Hou, Ionics, 2019, 25, 4057-4066.

12 C. A. Echeverria, W. Handoko, F. Pahlevani and V. Sahajwalla, J. Cleaner Prod., 2019, 208, 1524-1536.

13 H. Cheng, B. Gu, M. P. Pennefather, T. X. Nguyen, N. PhanThien and H. M. Duong, Mater. Des., 2017, 130, 452-458.

14 S. Huang and J. Shi, Ind. Eng. Chem. Res., 2014, 53, 48884893.

15 H. Bi, Z. Yin, X. Cao, X. Xie, C. Tan, X. Huang, B. Chen, F. Chen, Q. Yang and X. Bu, Adv. Mater., 2013, 25, 5916-5921.

16 Z. Y. Wu, C. Li, H. W. Liang, J. F. Chen and S. H. Yu, Angew. Chem., Int. Ed., 2013, 52, 2925-2929.

17 H. Chen, X. Wang, J. Li and X. Wang, J. Mater. Chem. A, 2015, 3, 6073-6081.

18 H. Chen, J. Li, X. Wu and X. Wang, Ind. Eng. Chem. Res., 2014, 53, 16051-16060.

19 S. Zhang, M. Zeng, J. Li, J. Li, J. Xu and X. Wang, J. Mater. Chem. A, 2014, 2, 4391-4397.

20 J. Zhu, H. Gu, J. Guo, M. Chen, H. Wei, Z. Luo, H. A. Colorado, N. Yerra, D. Ding, T. C. Ho, N. Haldolaarachchige, J. Hopper, D. P. Young, Z. Guo and S. Wei, J. Mater. Chem. A, 2014, 2, 2256-2265.

21 J. Ge, H. Y. Zhao, H. W. Zhu, J. Huang, L. A. Shi and S. H. Yu, Adv. Mater., 2016, 28, 10459.

22 P.-Y. Chen and S.-H. Tung, Macromolecules, 2017, 50, 25282534.

23 J. Saleem, M. A. Riaz and M. Gordon, J. Hazard. Mater., 2018, 341, 424-437.

24 A. L. Ahmad, S. Sumathi and B. H. Hameed, Chem. Eng. J., 2005, 108, 179-185.

25 W. Dai, S. J. Kim, W.-K. Seong, S. H. Kim, K.-R. Lee, H.-Y. Kim and M.-W. Moon, Sci. Rep., 2013, 3, 2524.

26 H. Liu, C.-Y. Cao, F.-F. Wei, P.-P. Huang, Y.-B. Sun, L. Jiang and W.-G. Song, J. Mater. Chem. A, 2014, 2, 3557-3562.

27 L. Jin, B. Hu, S. Kuddannaya, Y. Zhang, C. Li and Z. Wang, Polym. Compos., 2018, 39, E271-E277.

28 N. Xiao, Y. Zhou, Z. Ling and J. Qiu, Carbon, 2013, 59, 530536.

29 Y. Yang, Y. Deng, Z. Tong and C. Wang, J. Mater. Chem. A, 2014, 2, 9994-9999.

30 Z.-Q. Feng, F. Wu, L. Jin, T. Wang, W. Dong and J. Zheng, Ind. Eng. Chem. Res., 2019, 58, 3000-3008.
31 A. Stolz, S. Le Floch, L. Reinert, S. M. M. Ramos, J. TuaillonCombes, Y. Soneda, P. Chaudet, D. Baillis, N. Blanchard, L. Duclaux and A. San-Miguel, Carbon, 2016, 107, 198-208.

32 X. Gui, J. Wei, K. Wang, A. Cao, H. Zhu, Y. Jia, Q. Shu and D. Wu, Adv. Mater., 2010, 22, 617-621.

33 X. Ge, W. Yang, J. Wang, D. Long, L. Ling and W. Qiao, RSC $A d v .$, 2015, 5, 70025-70031.

34 N. Wang and Z. Deng, Mater. Res. Bull., 2019, 115, 19-26.

35 J. Zhao, W. Ren and H.-M. Cheng, J. Mater. Chem., 2012, 22, 20197.

36 Y. Meng, T. M. Young, P. Liu, C. I. Contescu, B. Huang and S. Wang, Cellulose, 2014, 22, 435-447.

37 W. Wan, R. Zhang, W. Li, H. Liu, Y. Lin, L. Li and Y. Zhou, Environ. Sci.: Nano, 2016, 3, 107-113.

38 H. Bi, X. Huang, X. Wu, X. Cao, C. Tan, Z. Yin, X. Lu, L. Sun and H. Zhang, Small, 2014, 10, 3544-3550.

39 J. Li, J. Li, H. Meng, S. Xie, B. Zhang, L. Li, H. Ma, J. Zhang and M. Yu, J. Mater. Chem. A, 2014, 2, 2934.

40 H. Wang, J. Zhu, X. Jin and H. Wu, J. Eng. Fibers Fabr., 2013, 8, 60-67.

41 Y. Lu, W. Wang, Y. Wang, M. Zhao, J. Lv, Y. Guo, Y. Zhang, R. Luo and X. Liu, Dalton Trans., 2018, 47, 16684-16695.

42 W. Leal Filho, D. Ellams, S. Han, D. Tyler, V. Boiten, A. Paco, H. Moora and A.-L. Balogun, J. Cleaner Prod., 2019, 218, 1020.

43 D. Angelova, I. Uzunov, S. Uzunova, A. Gigova and L. Minchev, Chem. Eng. J., 2011, 172, 306-311.

44 J. Ma, X. Wang, R. Gao, F. Zeng, C. Huang, P. Tontiwachwuthikul and Z. Liang, Fuel, 2016, 174, 296306.

45 M. M. Radetić, D. M. Jocić, P. M. Jovančić, Z. L. Petrović and H. F. Thomas, Environ. Sci. Technol., 2003, 37, 1008-1012.

46 O. Abdelwahab, Alexandria Eng. J., 2014, 53, 213-218.

47 W. Xie, Y. Li, W. Shi, L. Zhao, X. Zhao, P. Fang, F. Zheng and S. Wang, Chem. Eng. J., 2012, 213, 218-224.

48 H. Bi, X. Xie, K. Yin, Y. Zhou, S. Wan, L. He, F. Xu, F. Banhart, L. Sun and R. S. Ruoff, Adv. Funct. Mater., 2012, 22, 44214425.

49 Y. Zhao, C. Hu, Y. Hu, H. Cheng, G. Shi and L. Qu, Angew. Chem., Int. Ed., 2012, 51, 11174.

50 H. Sun, Z. Xu and C. Gao, Adv. Mater., 2013, 25, 2554-2560. 51 K. K. Gen Hayase, M. Fukuchi, H. Kaji and K. Nakanishi, Angew. Chem., Int. Ed., 2013, 52, 1986-1989.

52 J. Zhong, P. Wang, Y. Zhang, Y. Yan, S. Hu and J. Zhang, Energy, 2013, 59, 295-300.

53 A. Xie, J. Cui, Y. Chen, J. Lang, C. Li, Y. Yan and J. Dai, Sep. Purif. Technol., 2019, 215, 1-9.

54 Y. Shen, Q. Fang and B. Chen, Environ. Sci. Technol., 2014, 49, 67-84. 